ARTICLE

Received 29 May 2014 | Accepted 8 Oct 2014 | Published 2 Dec $2014 \quad$ DOl: 10.1038/ncomms6515

\title{
Mybpc3 gene therapy for neonatal cardiomyopathy enables long-term disease prevention in mice
}

Giulia Mearini ${ }^{1,2, \star}$, Doreen Stimpel ${ }^{1,2, \star}$, Birgit Geertz ${ }^{1,2}$, Florian Weinberger ${ }^{1,2}$, Elisabeth Krämer ${ }^{1,2}$, Saskia Schlossarek ${ }^{1,2}$, Julia Mourot-Filiatre ${ }^{1,2}$, Andrea Stoehr ${ }^{1,2}$, Alexander Dutsch ${ }^{1,2}$, Paul J.M. Wijnker ${ }^{1,2}$, Ingke Braren ${ }^{1,2,3}$, Hugo A. Katus ${ }^{4,5}$, Oliver J. Müller ${ }^{4,5}$, Thomas Voit ${ }^{6}$, Thomas Eschenhagen ${ }^{1,2}$ \& Lucie Carrier ${ }^{1,2}$

Homozygous or compound heterozygous frameshift mutations in MYBPC3 encoding cardiac myosin-binding protein $\mathrm{C}$ (cMyBP-C) cause neonatal hypertrophic cardiomyopathy (HCM), which rapidly evolves into systolic heart failure and death within the first year of life. Here we show successful long-term Mybpc3 gene therapy in homozygous Mybpc3-targeted knock-in (KI) mice, which genetically mimic these human neonatal cardiomyopathies. A single systemic administration of adeno-associated virus (AAV9)-Mybpc3 in 1-day-old KI mice prevents the development of cardiac hypertrophy and dysfunction for the observation period of 34 weeks and increases Mybpc3 messenger RNA (mRNA) and cMyBP-C protein levels in a dose-dependent manner. Importantly, Mybpc3 gene therapy unexpectedly also suppresses accumulation of mutant mRNAs. This study reports the first successful long-term gene therapy of HCM with correction of both haploinsufficiency and production of poison peptides. In the absence of alternative treatment options except heart transplantation, gene therapy could become a realistic treatment option for severe neonatal HCM.

\footnotetext{
${ }^{1}$ Department of Experimental Pharmacology and Toxicology, Cardiovascular Research Center, University Medical Center Hamburg-Eppendorf, 20246 Hamburg, Germany. ${ }^{2}$ DZHK (German Centre for Cardiovascular Research), Partner site Hamburg/Kiel/Lübeck, Hamburg, Germany. ${ }^{3}$ Hamburg Zentrum für Experimentelle Therapie Forschung (HEXT) Vector Core Unit, Department of Experimental Pharmacology and Toxicology, University Medical Center Hamburg-Eppendorf, 20246 Hamburg, Germany. ${ }^{4}$ Department of Cardiology, Internal Medicine III, University Hospital Heidelberg, Heidelberg, Germany. ${ }^{5}$ DZHK (German Centre for Cardiovascular Research), Partner site Heidelberg/Mannheim, 69120 Heidelberg, Germany. ${ }^{6}$ Université Pierre et Marie Curie UPMC-Inserm UMR S974, CNRS FRE 3617, Institut de Myologie, GH Pitié-Salpêtrière, Paris F-75013, France. * These authors contributed equally to this work. Correspondence and requests for materials should be addressed to L.C. (email: I.carrier@uke.de).
} 
ypertrophic cardiomyopathy (HCM) is the most prevalent inherited cardiac disease with an estimated frequency of 1:500 (ref. 1). HCM is transmitted in an autosomal dominant fashion (that is, one affected allele is sufficient to cause disease) and is caused by mutations in genes encoding components of the cardiac sarcomere. Out of them, MYBPC3 encoding cardiac myosin-binding protein C (cMyBP-C) is the most frequently mutated gene. More than $64 \%$ of $M Y B P C 3$ mutations are truncating, leading to unstable mutant polypeptides $^{2-5}$. Findings in humans as well as in cat and mouse HCM models indicate that the most prevalent disease mechanism is haploinsufficiency, even for MYBPC3 missense mutations ${ }^{6-8}$. Yet, mutant proteins ('poison peptides') may play an additional role in the pathogenesis of the disease ${ }^{9-11}$.

HCM is characterized by left ventricular hypertrophy (LVH) with or without left ventricular outflow tract obstruction and consequent congestion, diastolic dysfunction, myocardial disarray and increased interstitial fibrosis. HCM exhibits a large phenotypic variability. Whereas the mean life expectancy of patients who survived into young adulthood does not markedly differ from that of the normal population ${ }^{12}$, sudden cardiac death is common in young adults with HCM, particularly athletes ${ }^{13}$, and some patients develop severe systolic dysfunction and heart failure. Less known is that neonatal forms of HCM rapidly evolve into systolic heart failure and death within the first year of life $\mathrm{e}^{14-20}$. Some of these infants have homozygous or compound heterozygous frameshift MYBPC3 mutations ${ }^{14,16-20}$, expected to result in low level or absence of mutant cMyBP-C. Here we tested the easily generalizable idea to prevent the disease phenotype by adding full-length $M y b p c 3$ by gene therapy in a $M y b p c 3$-targeted knock-in (KI) mouse model ${ }^{21,22}$ that genetically mimics the severe neonatal HCM cases $^{21,22}$. These mice carry at the homozygous state the human c.772G > A MYBPC3 transition, which is one of the most frequent HCM mutations (13\% in a large Italian cohort $)^{23}$ and is associated with a bad prognosis ${ }^{19}$. Homozygous KI mice develop systolic dysfunction after postnatal day 1 and LVH at postnatal day 3 (refs 21,22), but are born with an apparently normal heart, providing the opportunity to treat prior to the onset of the disease phenotype.

In the present study, we administered a single dose of adenoassociated virus serotype 9 (AAV9)-Mybpc3 in 1-day-old KI mice. This prevents the development of cardiac hypertrophy and dysfunction and increases $M y b p c 3$ messenger RNA (mRNA) and cMyBP-C protein levels in a dose-dependent manner over a period of 34 weeks (wks). Importantly, $M y b p c 3$ gene therapy also suppresses accumulation of mutant mRNAs. This therapy could be a feasible choice for preventing the development of heart failure and death in neonatal cardiomyopathy.

\section{Results}

$M y b p c 3$ expression suppresses mutant mRNAs accumulation. KI mice carry a G $>$ A transition on the last nucleotide of exon 6 , which results in three different mutant mRNAs (Fig. 1a) and a $>80 \%$ reduction of total $M y b p c 3$ mRNA and cMyBP-C protein levels ${ }^{8}$. We first evaluated the level of expression of FLAG-tagged $M y b p c 3$ after gene transfer in cardiac myocytes isolated from KI neonatal mice. Cardiac myocytes were transduced with AAV serotype 6 (AAV6) encoding FLAG-tagged cMyBP-C or green fluorescent protein (GFP) (AAV6-Mybpc3 or AAV6-GFP), under the control of the human cardiac troponin T promoter (TNNT2; Fig. 1b) for 7 days. Whereas cMyBP-C protein level was low $(<20 \%$ of wild-type (WT)) in non-transduced or GFP-transduced KI cardiac myocytes, it increased to 70\% after AAV6-Mybpc3 gene transfer (Fig. 2a). FLAG-cMyBP-C protein was detected in $83 \%$ of cMyBP-C-positive cells and was correctly incorporated in doublets in the A-band of the sarcomere (Fig. 2b).
We then evaluated whether AAV6-Mybpc3 gene transfer prevented a KI disease phenotype in three-dimensional engineered heart tissue (EHT) generated from neonatal mice. As shown earlier, KI EHTs exhibit higher sensitivity to external $\mathrm{Ca}^{2}+$ and higher mRNA levels of hypertrophic markers than WT $\mathrm{EHTs}^{24}$. As expected, force of contraction and velocity of both contraction and relaxation were higher in non-transduced KI than WT EHTs (Fig. 2c) and were not affected by AAV6-GFP. On the other hand, all parameters were lower in AAV6-Mybpc3transduced KI EHTs than in non-transduced KI, almost reaching values of non-transduced WT EHTs. This suggests that AAV6Mybpc3 gene transfer prevented the KI EHTs phenotype ( = reduction of hypercontractility). Reverse transcription (RT)PCR using specific primers (Supplementary Table 1) revealed a FLAG-Mybpc3 mRNA product only in KI EHTs transduced with AAV6-Mybpc3, but not in the other EHTs (Fig. 2d). Analysis of total Mybpc3 mRNA using common primers revealed mutant-1, mutant- 2 and mutant-3 mRNAs (Fig. 1a) in non-transduced KI EHTs (Fig. 2d). In contrast, AAV6-Mybpc3-transduced EHTs exhibited mainly a single mRNA PCR product, similar to WT EHTs (Fig. 2d). RT-quantitative (q)PCR with specific Taqman probes (Fig. 1d; Supplementary Table 1) performed in pooled RNAs confirmed a marked accumulation of WT mRNAs and disappearance of missense (mutant-1) and frameshift (mutant-2 and mutant-3) mRNAs in EHTs transduced with AAV6-Mybpc3 (Fig. 2e,f). This suggests that exogenous $M y b p c 3$ expression unexpectedly prevented accumulation of mutant mRNAs in KI EHTs. Moreover, AAV6-Mybpc3 transduction reduced elevated mRNA levels of hypertrophy markers in KI EHTs (Fig. 2g). These data suggest that ex vivo Mybpc3 gene therapy prevents both the molecular and functional disease phenotype of KI EHTs.

Long-term prevention of LVH and dysfunction by gene therapy. Next we evaluated whether $M y b p c 3$ gene therapy could prevent the molecular and functional disease phenotype of $\mathrm{KI}$ mice in vivo. KI mice are born without a disease phenotype, but develop reduced fractional area shortening (FAS) between days 1 and 2 and increased left ventricular mass-to-body-weight ratio (LVM/BW) at postnatal days 3-4 (refs 21,22). Escalating doses of AAV9-Mybpc3 $\left(1 \times 10^{11}, 3 \times 10^{11}, 1 \times 10^{12}\right.$ and $3 \times 10^{12}$ vector genomes (vg)) or phosphate-buffered saline (PBS) were administered into the temporal vein ${ }^{21,22,25}$ of 1 -day-old KI mice (Fig. 1c). A total of 10 mice per AAV9 dose (except for the $1 \times 10^{11}$ dose, $n=6$ ) were included in the study (Supplementary Table 2). Some pups treated with AAV9 died during the first weeks, which was independent of the dose administered, but was related to some mothers eating their pups. A total of 5-7 mice treated with AAV9 per group remained and were compared with $12 \mathrm{KI}-\mathrm{PBS}$ and $10 \mathrm{WT}$ mice. Cardiac function was evaluated by serial echocardiography at 2,12, 16, 25 and 34 wks of age (Fig. 1c). As expected, PBS-injected KI mice had already an almost two-fold higher LVM/BW ratio at 2 wks of age, which was due to more than two-fold higher LVM despite the $20 \%$ higher BW, and $32 \%$ lower FAS than WT (Fig. 3a-d). BW was higher and independent of the treatments in all KI than WT mice (Fig. 3b), indicating that KI mice gained weight quicker than WT mice. AAV9-Mybpc3 prevented the increase in LVM/BW ratio in a dose-dependent manner (Fig. 3c). Whereas AAV9 doses of $1 \times 10^{11}$ and $3 \times 10^{11} \mathrm{vg}$ did not have an apparent therapeutic effect, KI mice treated with the highest dose did not differ from WT, but significantly differed from KI-PBS mice. Although the therapeutic effect of $M y b p c 3$ gene therapy on FAS was less marked than that on LVM/BW, there was also a dose-dependent trend towards prevention of cardiac dysfunction at 2 wks of age (Fig. 3d; Supplementary Table 3). Similar results were observed 
a

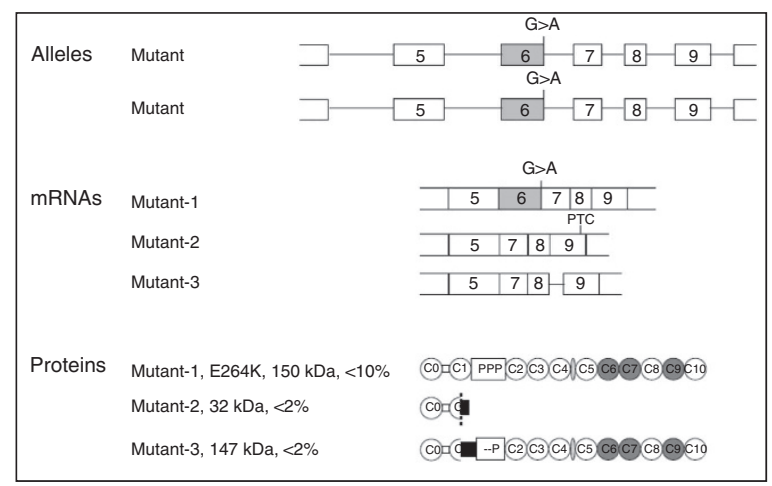

d

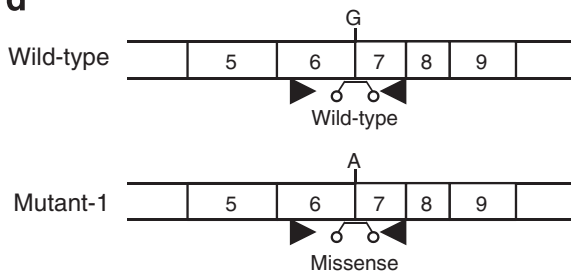

Mutant-2

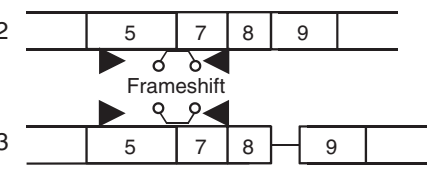

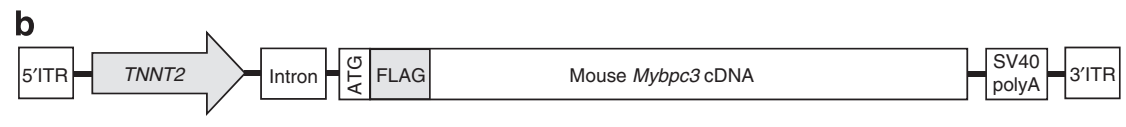

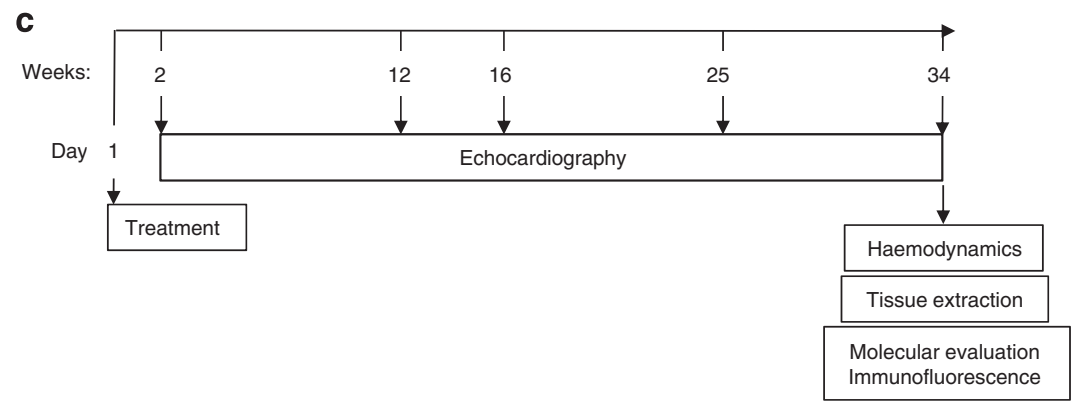

Figure 1 | Schematic representation of the molecular consequence of the KI mutation, the vector used, protocol for Mybpc3 gene therapy and location of primers and probes. (a) Consequence of the G>A transition on the last nucleotide of Mybpc3 exon 6 at mRNA and protein levels in homozygous Mybpc3-targeted knock-in (KI) mice. Mutant-1 mRNA (missense) contains the G>A transition, expected to produce an E264K 150-kDa cMyBP-C. Mutant2 (nonsense) and mutant-3 (deletion/insertion) mRNAs result from exon-6 skipping. Mutant-2 contains a premature termination codon (PTC) in exon 9 and is expected to produce a 32-kDa truncated cMyBP-C. Mutant-3 mRNA retains parts of intron 8 , which restores the reading frame, and should produce a 147-kDa cMyBP-C. (b) Schematic linear representation of the pGG2 vector expressing FLAG-tagged mouse wild-type Mybpc3 cDNA under the control of the human cardiac troponin T promoter (TNNT2). (c) Experimental protocol in vivo. Adeno-associated virus serotype 9 (AAV9)-Mybpc3 (1 $\times 10^{11}, 3 \times 10^{11}$, $1 \times 10^{12}$ or $3 \times 10^{12}$ vector genomes) or PBS was systemically administered in 1-day-old KI mice and compared with wild-type mice. Echocardiography was performed at different postnatal time points until $34 \mathrm{wks}$ of age. At the end of the protocol, mice were subjected to haemodynamics, tissues were extracted and evaluated for molecular biology (RNA and proteins) and for immunofluorescence (cardiac sections). (d) Location of primers (arrows) and Taqman-specific probes (between primers) in wild-type and mutant Mybpc3 mRNA structures.

12, 16 and 25 wks after treatment (Supplementary Table 3). Longterm effect of AAV9-Mybpc3 gene therapy was eventually evaluated 34 wks post administration and exhibited dosedependent prevention of the increase in LVM/BW and decrease in FAS (Fig. 3e-h; Supplementary Table 3).

To evaluate contractile function with a second method, mice were subjected to haemodynamic measurements by LV catheterization before killing at $34 \mathrm{wks}$ of age (Figs 1c and 4). Some of the mice, independently of the genotype or treatment, could not be analysed due to technical problems (for example, intubation, ventilation or bleeding). Systolic function $\left(\mathrm{d} P / \mathrm{d} t_{\max }\right)$ was slightly, but non-significantly lower in PBS-treated KI than in WT mice $(P<0.06$, Student's $t$-test; Fig. $4 \mathrm{a})$. Whereas $\mathrm{d} P / \mathrm{d} t_{\max }$ did not differ between KI-3 $\times 10^{11} \mathrm{vg}$ and KI-PBS, it was higher in KI treated with the two highest doses of AAV9-Mybpc3 than KI-PBS $(P<0.05$, Student's $t$-test $)$ and did not differ from WT mice $(P=0.6$, Student's $t$-test; Fig. $4 \mathrm{a})$. Diastolic function $\left(\mathrm{d} P / \mathrm{d} t_{\min }\right)$ was markedly lower $(-44 \%)$ in PBS-treated KI, and this reduction was partially prevented in KI mice treated with the two highest doses of AAV9-Mybpc3 ( $P=0.02$ and 0.07 versus WT, respectively, Student's $t$-test; Fig. $4 \mathrm{~b}$ ). Heart weight (HW) to BW (HW/BW) and HW-to-tibia-length (HW/TL) ratios as measured after killing in all animals were $52-55 \%$ higher in PBS-treated KI than in WT mice (Fig. 4c,d). Mybpc3 gene therapy induced a dose-dependent reduction in $\mathrm{HW} / \mathrm{BW}$ and $\mathrm{HW} / \mathrm{TL}$ ratios, confirming the echocardiographic data with less intragroup variability. The two highest doses groups did not differ from WT.

To assess the average level of $M y b p c 3$ overexpression, pools of ventricular RNA and proteins were made by combining identical amounts from all 34-wk-old KI and WT mice. Exogenous FLAG$M y b p c 3$ mRNA was detected only in mice that received AAV9$M y b p c 3$, and its level increased with increasing AAV9-Mybpc3 dose (Fig. 5a). Mybpc3 gene therapy also dose-dependently increased the level of total full-length $M y b p c 3$ mRNA, and, similar to the effect observed in EHTs, reduced the level of mutant Mybpc3 mRNAs (Fig. 5a). At the highest AAV9 dose $\left(=3 \times 10^{12} \mathrm{vg}\right)$, mutant $M y b p c 3$ mRNAs were barely detected. Total Mybpc3 mRNA level in KI-PBS mice represented $15 \%$ of the WT mouse level (Fig. 5b). This level did not differ with the first three AAV9 doses, whereas the highest AAV9-Mybpc3 dose almost fully restored the amount of total $M y b p c 3$ mRNA to the WT level (Fig. 5b). The levels of WT and mutant Mybpc3 mRNAs were then determined by RT-qPCR with specific Taqman probes. 
a

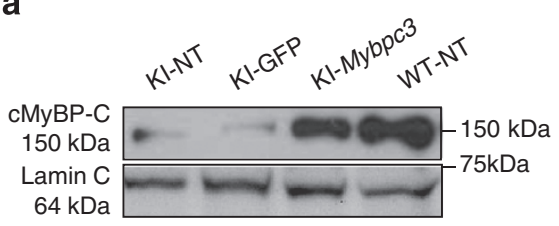

b

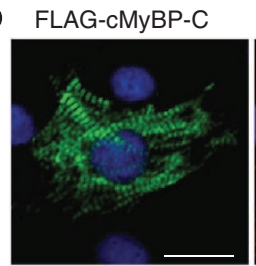

C
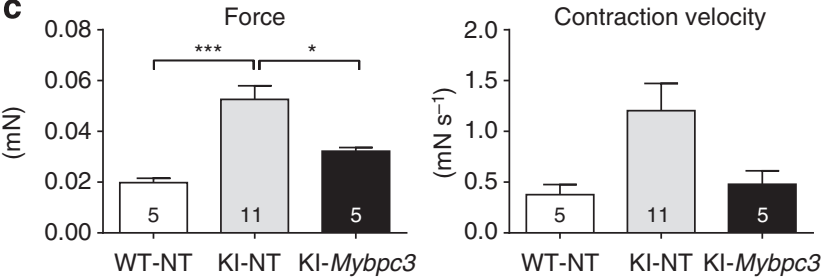
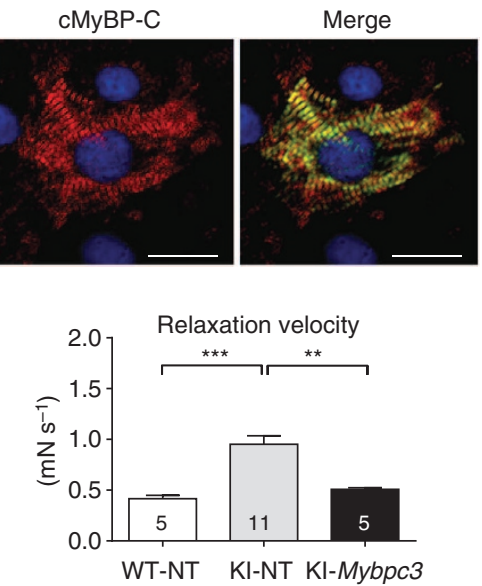

d
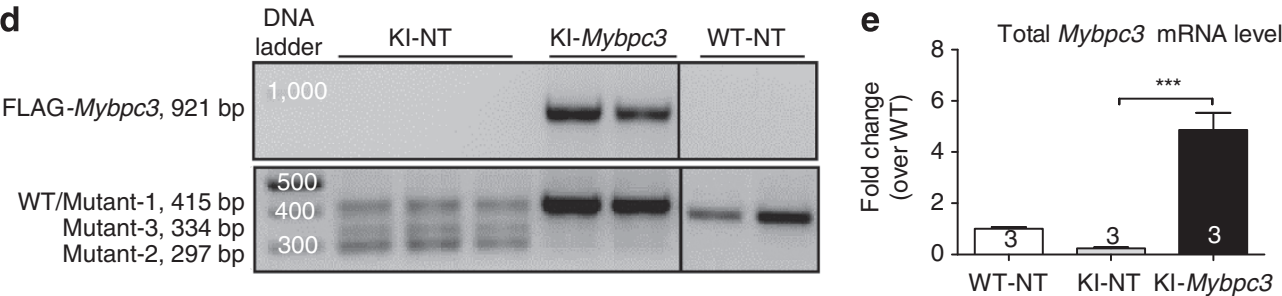

f
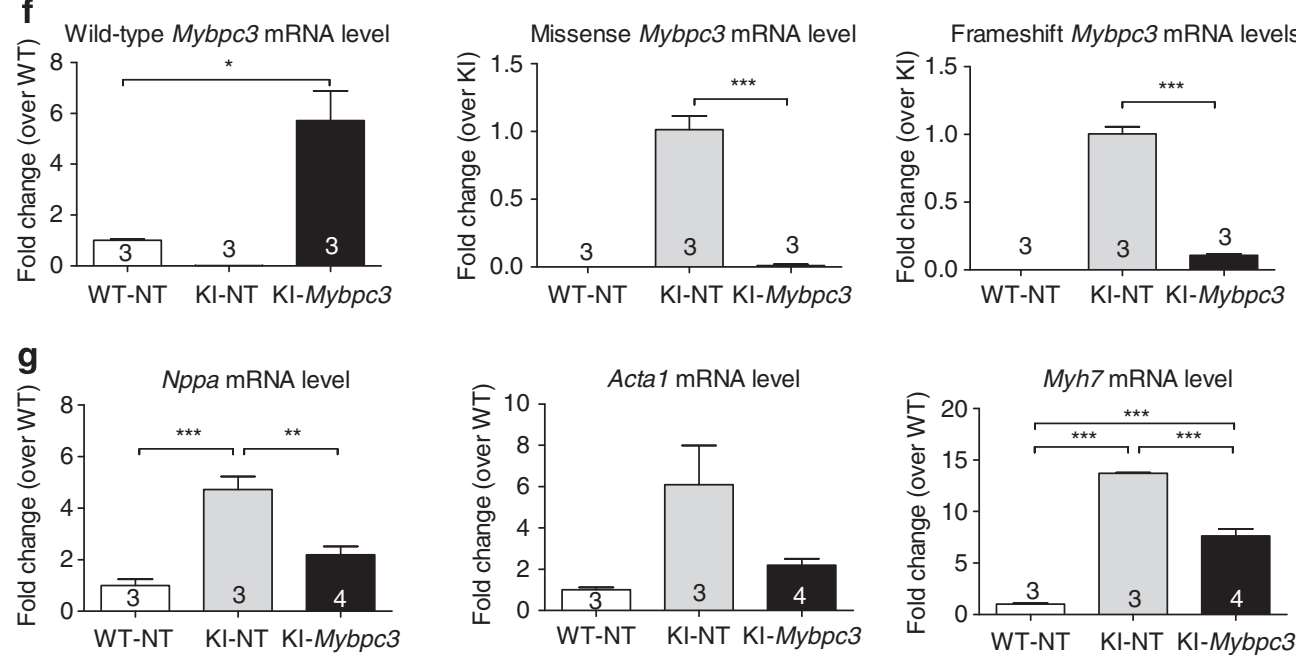

Figure 2 | Impact of Mybpc3 gene transfer in cardiac myocytes in two-dimensional (2D) culture or three-dimensional (3D) engineered heart tissue. Cardiac myocytes were isolated from WTor KI neonatal mice, and were immediately transduced or not with AAV6 encoding FLAG-cMyBP-C or GFP under the control of human TNNT2 promoter at a MOI of 3,000 for 7 days in 2D culture (a,b) or at a MOI of 1,000 for 19 days in 3D engineered heart tissue (c-g). (a) Western blot performed on protein extracts of cardiac myocytes stained with anti-cMyBP-C and anti-lamin A/C antibodies. (b) Immunofluorescence analysis of AAV6-Mybpc3-transduced KI cardiac myocytes. Cells were double-stained with anti-FLAG and anti-cMyBP-C antibodies. Nuclei were stained with DRAQ5. Scale bar, $20 \mu \mathrm{m}$. (c) Contractile function of spontaneously beating EHTs measured with video-optical recording. Force, contraction velocity and relaxation velocity were measured at day 19 in culture medium containing $1.8 \mathrm{mM} \mathrm{Ca}^{2+}$. Number of EHTs is indicated in the bars. (d) RT-PCR of exogenous (FLAG-Mybpc3) and total Mybpc3 (including WT and/or mutants) mRNAs amplified with specific and common primers, respectively, in RNA from Mybpc3-transduced KI and KI-NT and WT-NT EHTs. Amplicon size is shown on the left side. (e) Total Mybpc3 mRNA level determined by RT-qPCR with SYBR Green in RNA from WT-NT, KI-NT and KI-Mybpc3 EHTs. (f) Levels of wild-type, missense and frameshift (mutant-2 + mutant-3) mRNAs in RNA from WT-NT, KI-NT and KI-Mybpc3 EHTs determined with specific hydrolysis Taqman probes. (g) mRNA levels of hypertrophic markers determined by RT-qPCR. All mRNAs were normalized to G $\alpha$ S. Data are expressed as mean \pm s.e.m. ${ }^{\star} P<0.05$, ${ }^{\star \star} P<0.01$ and ${ }^{\star \star \star} P<0.001$, one-way ANOVA plus Bonferroni's post-test (in $\mathbf{e}, \mathbf{g}$ ) or Student's $t$-test (in $\mathbf{f}$ ). For mRNA analysis, 3-4 EHTs per group were included. Each qPCR was done in triplicate. Acta1, $\alpha$-skeletal actin; EHT, engineered heart tissue; KI, knock-in; MOI, multiplicity of infection; Myh7, $\beta$-myosin heavy chain; Nppa, atrial natriuretic factor; NT, non-transduced; WT, wild-type.

Whereas WT mRNA level dose-dependently increased in KI up to $64 \%$ of the level found in WT mice, missense and frameshift mRNA levels dose-dependently decreased simultaneously (Fig. 5c). With the highest AAV9 dose, missense mRNA was fully suppressed and frameshift mRNA decreased by $33 \%$, resulting in the estimation that $<6 \%$ of total mRNAs is frameshift and the rest is WT. Similar to the mRNA level, the level of exogenous FLAG-cMyBP-C protein increased with increasing AAV9-Mybpc3 doses in pooled proteins (Fig. 5d). The level of full-length cMyBP-C protein normalized to glyceraldehyde 3-phosphate dehydrogenase $(\mathrm{GAPDH})$ in AAV9-Mybpc3-treated KI with the highest dose reached $57 \%$ of 
a
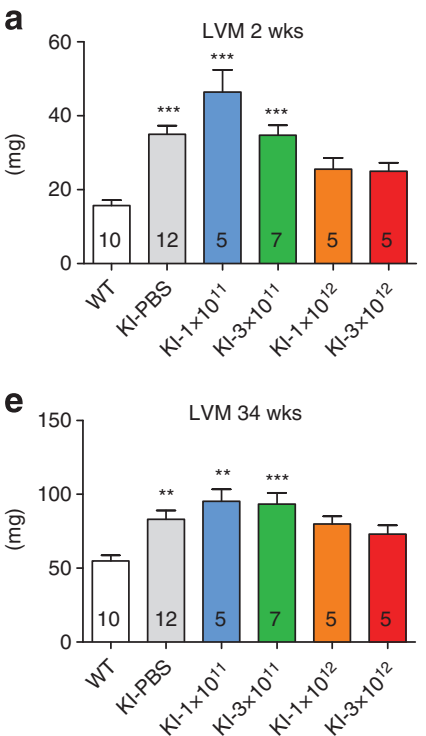

b

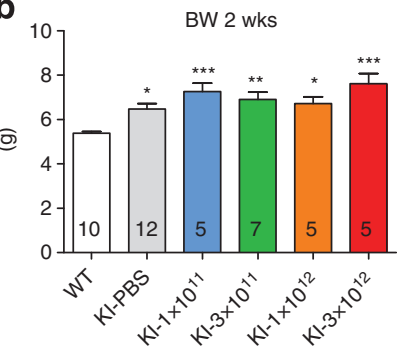

f

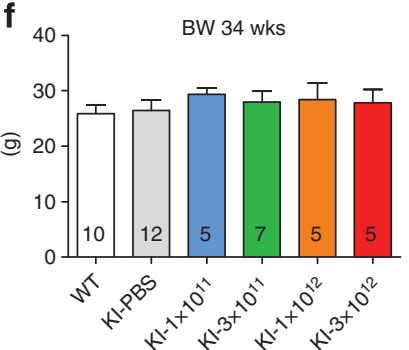

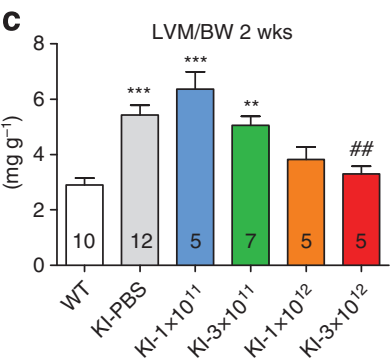
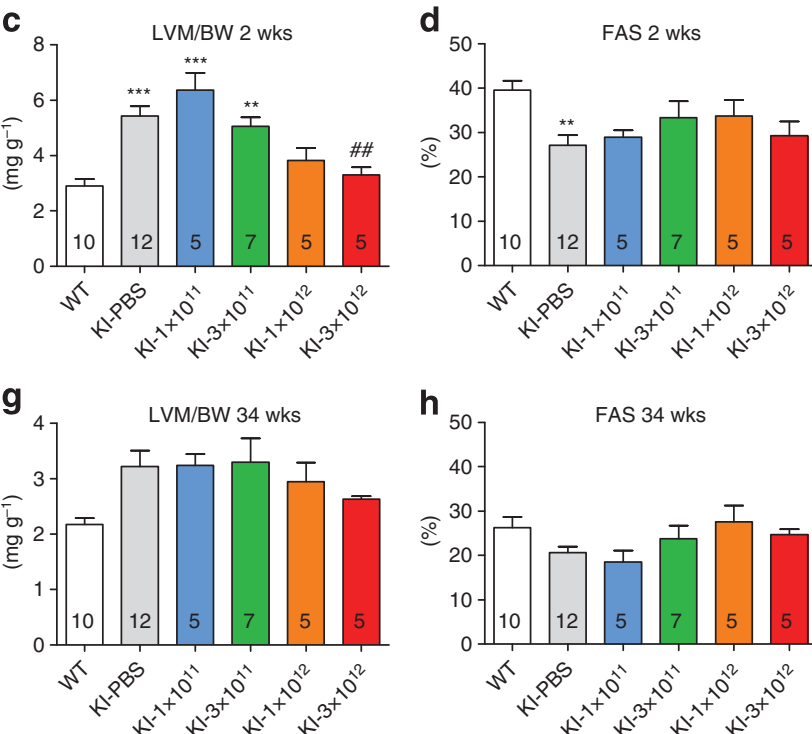

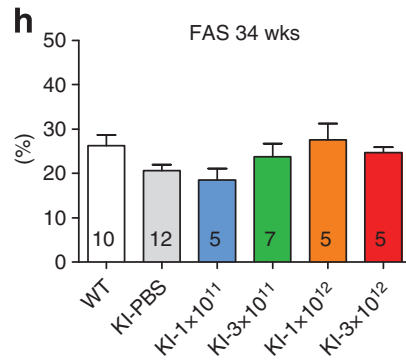

Figure 3 | Impact of gene therapy in vivo-evaluation of cardiac phenotype by echocardiography. AAV9 $\left(1 \times 10^{11}, 3 \times 10^{11}, 1 \times 10^{12}\right.$ or $3 \times 10^{12}$ vector genomes) encoding FLAG-cMyBP-C was systemically administered via the temporal vein in 1-day-old $\mathrm{KI}$ mice and compared with age-matched PBS-treated KI and WT mice 2 wks (a-d) and 34 wks (e-h) post AAV9 administration. (a,e) Left ventricular mass (LVM). (b,f) Body weight (BW). $(\mathbf{c}, \mathbf{g})$ Left ventricular mass-to-body-weight ratio (LVM/BW). (d,h) Fractional area shortening (FAS). Values are mean \pm s.e.m. ${ }^{\star} P<0.05,{ }^{\star \star} P<0.01$, ${ }^{\star \star \star} P<0.001$ versus WT and ${ }^{\# \#} P<0.01$ versus KI-PBS, one-way ANOVA plus Bonferroni's post-test. Number of mice is indicated in the bars.
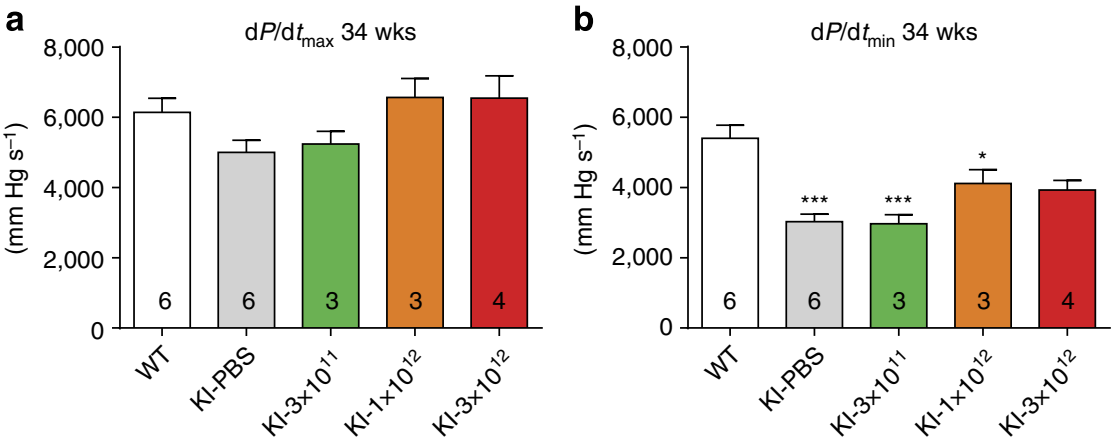

C

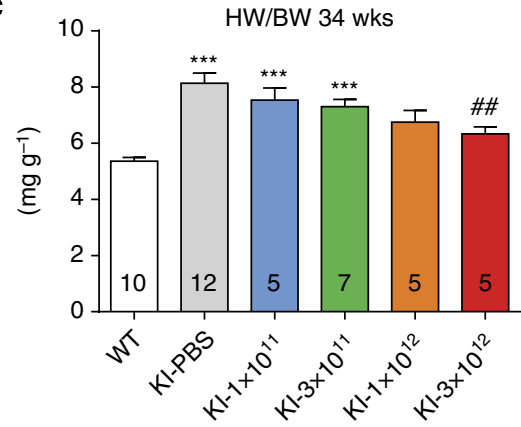

d

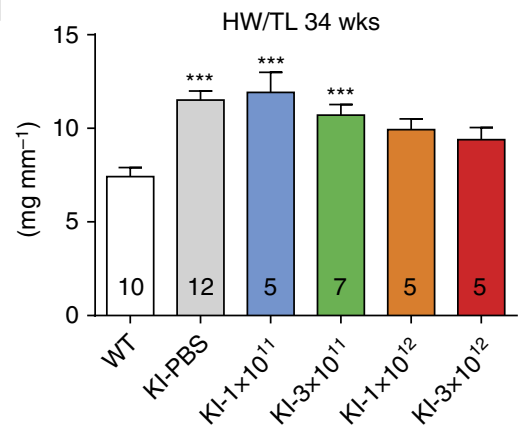

Figure 4 | Haemodynamic measurements and cardiac hypertrophy determination 34 weeks after AAV9-Mybpc3 administration. AAV9-Mybpc3 $\left(1 \times 10^{11}, 3 \times 10^{11}, 1 \times 10^{12}\right.$ or $3 \times 10^{12}$ vector genomes)-treated KI mice were compared with age-matched PBS-treated KI and WT mice. (a) Systolic function $\left(\mathrm{d} P / \mathrm{d} t_{\max }\right)$. (b) Diastolic function $\left(\mathrm{d} P / \mathrm{d} t_{\min }\right)$. (c) Heart weight to body weight ratio $(\mathrm{HW} / \mathrm{BW})$. (d) Heart weight to tibia length ratio $(\mathrm{HW} / \mathrm{TL})$. Values are mean \pm s.e.m. ${ }^{\star} P<0.05$ and ${ }^{\star \star \star} P<0.001$ versus WT, ${ }^{\# \#} P<0.01$ versus KI-PBS, one-way ANOVA plus Bonferroni's post-test. Number of mice is indicated in the bars. The mice that received $1 \times 10^{11}$ vector genomes were not analysed by haemodynamics.

that in WT mice. These data in pooled samples were supported by statistical analyses of individual samples $(=59 \%$ of that in WT, Fig. 5e).

To assess whether AAV9-mediated gene therapy with a cardiac-specific promoter carries the risk of off-target effects in other organs, we took tissue samples from liver and skeletal muscle of one of the AAV9-Mybpc3-treated KI mice treated with the highest dose and subjected them to RT-PCR and western blot. FLAG-Mybpc3 mRNA was amplified in ventricles, and to a very low extent in liver or skeletal muscle (Fig. 5f). FLAG-cMyBP-C protein was only detected in the ventricles, but not in liver and skeletal muscle (Fig. 5g). 
a
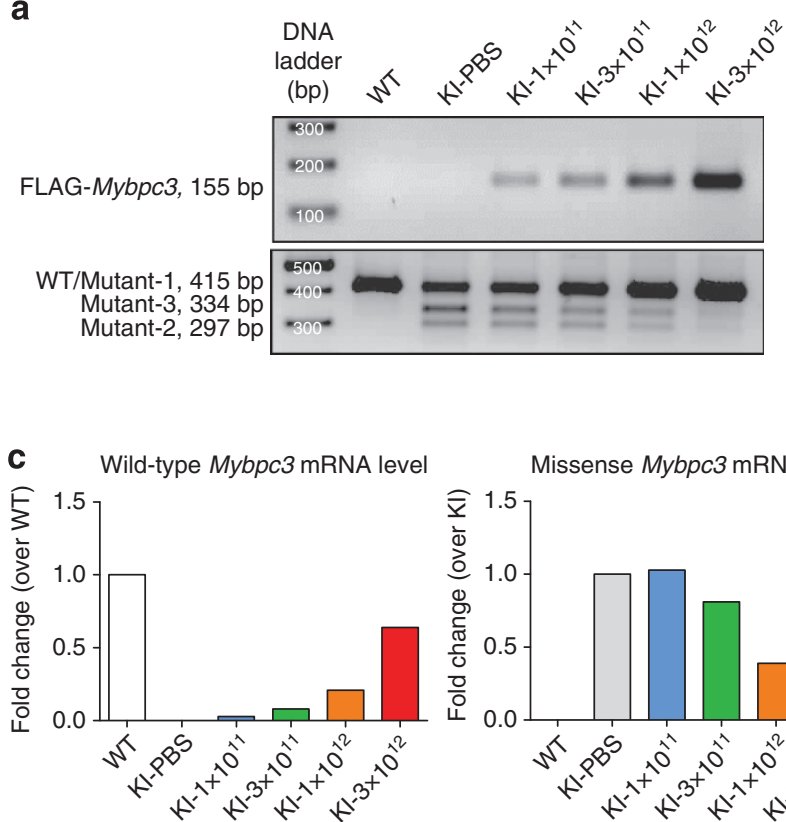

d

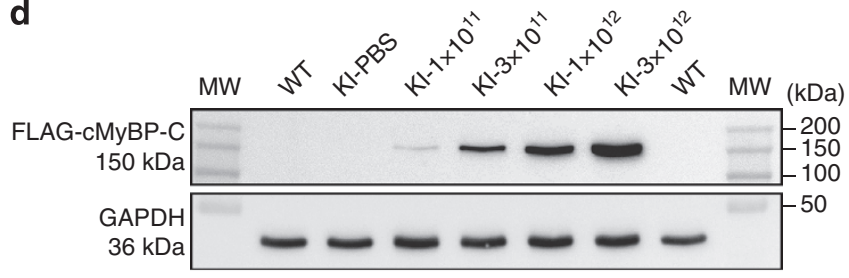

e

Missense Mybpc3 mRNA level

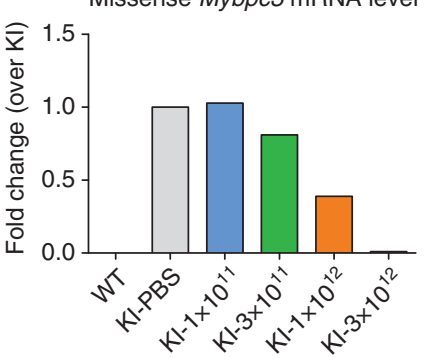

b

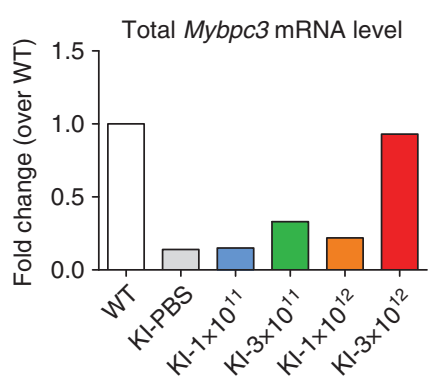

Frameshift Mybpc3 mRNA level
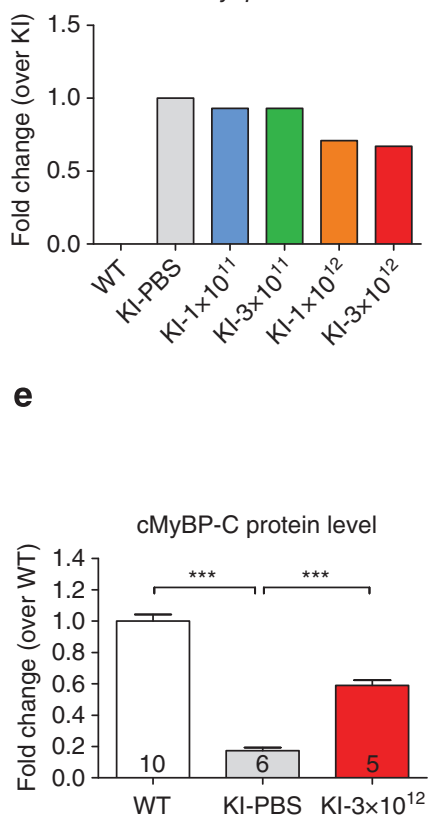

f

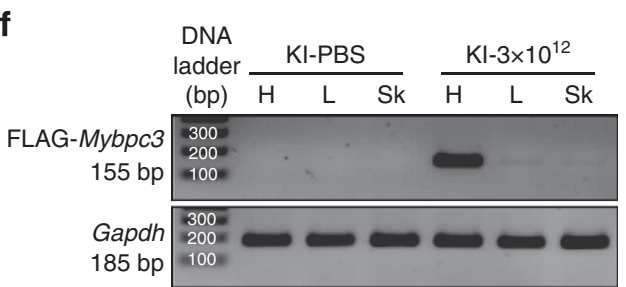

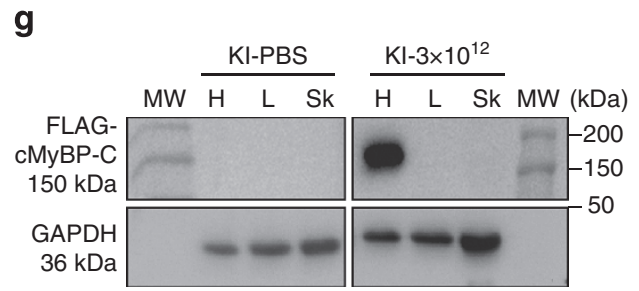

Figure 5 | Evaluation of Mybpc3 expression after AAV9-mediated Mybpc3 gene therapy in vivo. Ventricular tissues were extracted from 34-wks-old KI and WT mice that received or not AAV9 $\left(1 \times 10^{11}, 3 \times 10^{11}, 1 \times 10^{12}\right.$ or $3 \times 10^{12}$ vector genomes) encoding FLAG-cMyBP-C. (a) RT-PCR of exogenous (FLAG-Mybpc3) and total Mybpc3 (including WT and/or mutants) mRNAs amplified with specific and common primers in pooled ventricular RNA, respectively ( $n=5-10$ per group). Amplicon sizes are shown on the left side. (b) Total Mybpc3 mRNA level determined by RT-qPCR with SYBR Green in pooled ventricular RNA from all groups. (c) Levels of wild-type, missense and frameshift (mutant-2 + mutant-3) Mybpc3 mRNAs determined with specific hydrolysis Taqman probes in pooled ventricular RNA from all groups. (d) Western blots performed in pooled ventricular protein extracts ( $n=5-10$ per group) were stained with antibodies directed against exogenous FLAG-cMyBP-C, total CMyBP-C or endogenous glyceraldehyde 3-phosphate dehydrogenase (GAPDH). (e) Quantification of cMyBP-C protein level in individual samples from WT, KI-PBS and KI-3 $\times 10^{12}$, normalized to Ponceau and related to WT. (f) RT-PCR of FLAG-Mybpc3 and Gapdh in heart $(\mathrm{H})$, liver (L), skeletal muscle (Sk) of KI-PBS and KI-3 $\times 10^{12}$ mice. MW of the amplicons is shown on the left side. (g) Western blots performed with antibodies directed against exogenous FLAG-cMyBP-C or GAPDH in heart (H), liver (L), skeletal muscle (Sk) of KI-PBS and KI-3 $\times 10^{12}$ mice. Values are mean \pm s.e.m. ${ }^{\star \star \star} P<0.001$, one-way ANOVA plus Dunnett's post-test versus KI-PBS. Number of mice is indicated in the bars. Each qPCR was performed in triplicate. KI, knock-in; MW, molecular weight markers; WT, wild-type.

Evaluation of cardiac sections by immunofluorescence showed exogenous FLAG-cMyBP-C protein to be organized in doublets in the A-band of the sarcomere and in alternation with $\alpha$-actinin, indicating correct sarcomeric incorporation (Fig. 6a). Of note, not all $\alpha$-actinin-positive cardiac myocytes were FLAG-positive, suggesting incomplete transduction. A cMyBP-C antibody recognizing both endogenous and exogenous cMyBP-C proteins showed typical A-band doublets throughout the section in WT mice and a much weaker, less-defined signal in PBS-treated KI, reflecting the lower total cMyBP-C protein amount in KI 
a
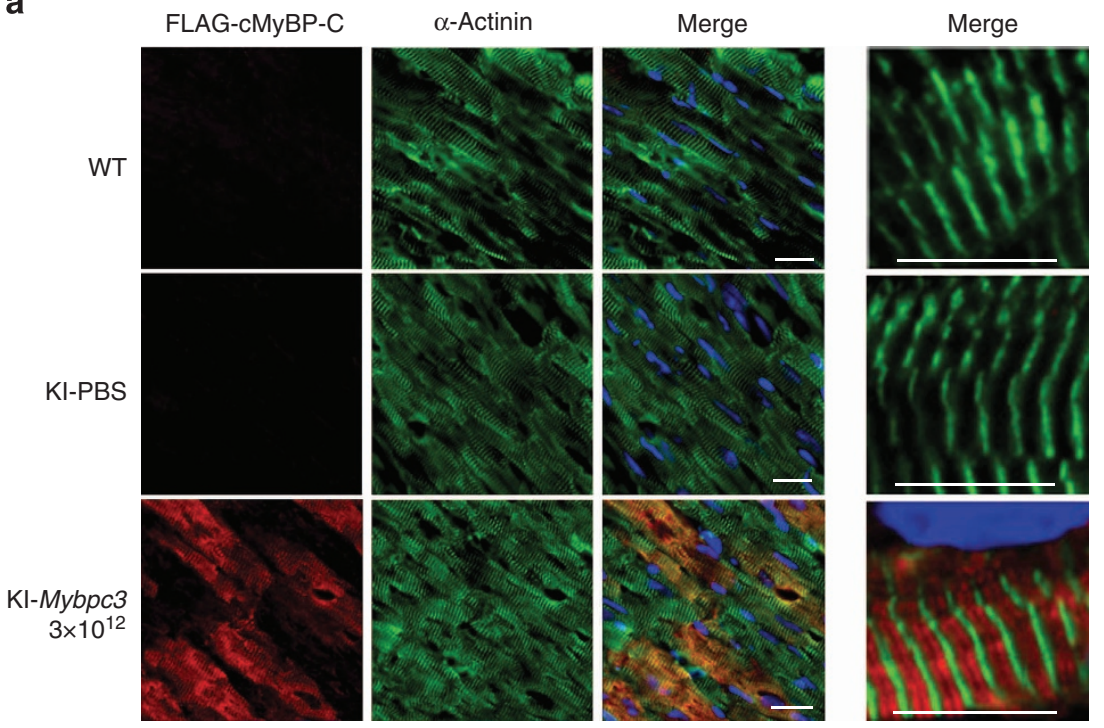

b
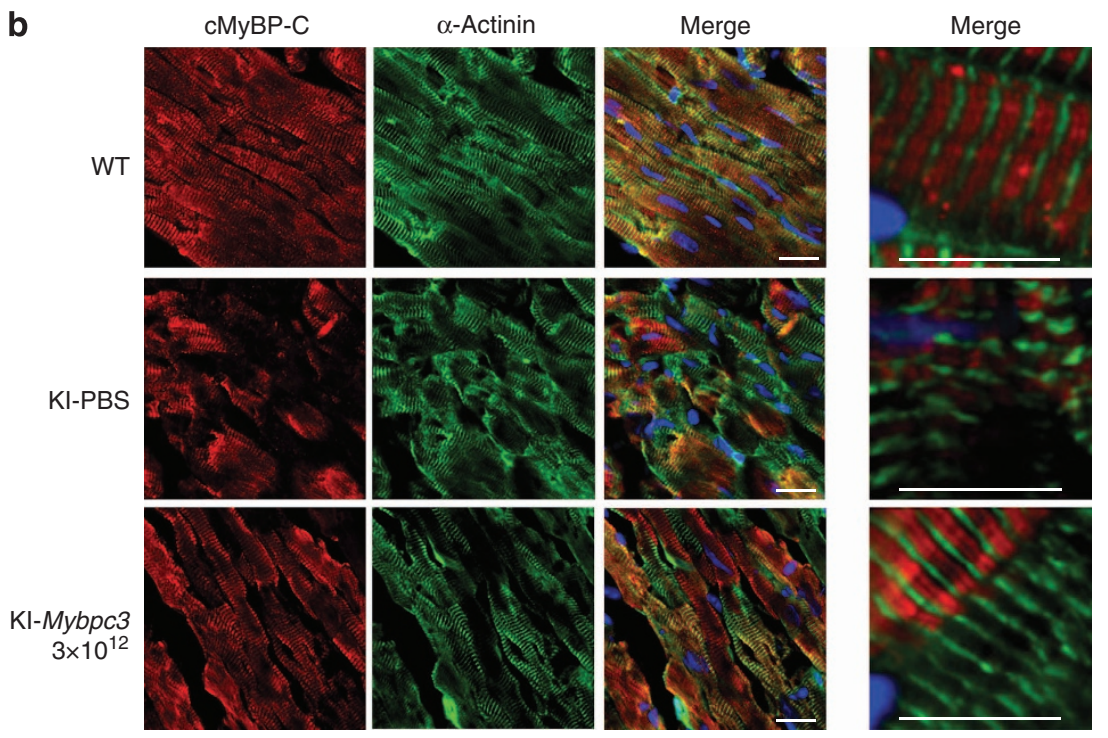

Figure 6 | Immunofluorescence analysis of cardiac sections 34 weeks after AAV9-Mybpc3 administration. Hearts from WT, KI treated with PBS or with $3 \times 10^{12}$ virus genomes of AAV9-Mybpc3 were extracted and cryosections were performed. (a) Double-staining of cardiac sections with anti-FLAG and anti- $\alpha$-actinin antibodies. (b) Double-staining of cardiac sections with anti-cMyBP-C and anti- $\alpha$-actinin antibodies. Nuclei were stained with DRAQ5. Scale bar, $20 \mu \mathrm{m}$. Merge images on the right panel were obtained at a higher magnification (scale bar, $10 \mu \mathrm{m}$ ).

(Fig. 6b). The signal was much stronger in AAV9-Mybpc3-treated $\mathrm{KI}$, but again, not all cardiac myocytes were stained.

\section{Discussion}

This study reports the first successful long-term gene therapy of HCM, the most common inherited cardiac disease. Several aspects are notable: (i) AAV-mediated $M y b p c 3$ expression not only corrected the reduction of total cMyBP-C mRNA and protein levels as expected, but also suppressed the production of mutant mRNA species, which may give rise to pathological proteins. Thus, Mybpc3 gene therapy appears to correct both haploinsufficiency and poison peptide mechanisms as underlying causes of HCM. (ii) In fact, our $M y b p c 3$ gene therapy approach was functionally successful in a KI mouse model of HCM, which exhibits both the reduction of total mRNA/protein levels and pathological mRNA species. (iii) The prevention of the disease phenotype by gene therapy was clearly AAV9-dose-dependent, almost complete and long lasting. (iv) By principle, $M y b p c 3$ gene therapy, in contrast to mutation-specific therapy approaches, should be applicable to all forms of HCM caused by MYBPC3 gene mutations. Thus, the present data are an important step towards clinical translation of gene therapy for patients with severe, MYBPC3-related forms of HCM.

Gene therapy for HCM comes in the continuity of gene therapy for heart failure, targeting proteins involved in calciumhandling such as phospholamban ${ }^{26}$ and S100A1 (ref. 27). The recent successful completion of phase II trials of SERCA2a gene therapy demonstrated the feasibility and safety of AAV1mediated gene transfer as well as improvement of the symptoms and exercise capacity of patients with advanced heart failure ${ }^{28}$. Classical treatments of HCM target symptoms and LV outflow tract obstruction with pharmacological and/or surgical treatments, but do not address the cause of the disease ${ }^{29,30}$. New strategies targeting the endogenous mutant pre-mRNA or RNA such as exon skipping, exon inclusion, trans-splicing and 
RNA silencing, opened the perspective for a causal therapy for patients with $\mathrm{HCM}^{2,21,22,31,32}$. These proof-of-concept studies still have major limitations. We previously showed that a $5^{\prime}$-transsplicing approach corrects the mutation in cardiac myocytes and in vivo in HCM KI mice, but its efficiency was too low to prevent or rescue the disease phenotype ${ }^{22}$. In-frame skipping of mutated exons by antisense oligonucleotides inserted in U7snRNA produced a stable functional protein and transiently rescued the cardiac phenotype in KI mice ${ }^{21}$. In both cases, however, the amount of repaired protein remained very $l_{0}{ }^{21,22}$. Another RNA-targeting approach, the allele-specific silencing using oligonucleotides has been recently evaluated in another HCM mouse model carrying a mutation in Myh6 encoding $\alpha$-myosin heavy chain showing disease prevention until 25 wks of age, which, in this model, needs to be revealed by application of cyclosporine ${ }^{31}$.

The present $M y b p c 3$ gene therapy approach was not a priori considered as a feasible therapy strategy for HCM for two reasons. First, it is generally assumed that the packaging capacity of AAV is limited to $\sim 4.9 \mathrm{kbp}$, while longer sequences have been shown to lead to truncation of AAV particles ${ }^{33}$ or to markedly decrease transduction efficiency ${ }^{34}$. However, we demonstrate herein that packaging of an oversized DNA sequence of $5.4 \mathrm{kbp}$ (including two inverted terminal repeats, the human TNNT2 promoter, a chimeric intron, the FLAG-tagged Mybpc3 complementary DNA (cDNA) and the SV40 polyadenylation signal) was compatible with efficient production of AAV6 and AAV9, and that the vectors induced efficient expression of fulllength $M y b p c 3$ in isolated mouse cardiac myocytes and EHTs, as well as in the mouse heart in vivo. Second, it was not expected that expression of the WT protein also prevents the production of mutant mRNAs. Yet, this was the case both ex vivo and in vivo, and this was dose-dependent. The molecular mechanism is not clear at present, but the paracrystalline structure of sarcomeric scaffolding suggests that sarcomeric proteins, in particular those contributing to the contractile mechanism such as actins and myosins, are in a precise stoichiometric equilibrium ${ }^{35}$. As AAVinduced WT cMyBP-C protein would be more stably integrated into the sarcomere, yet unknown feedback mechanisms might downregulate transcription of the mutant allele. If this hypothesis was true, gene therapy could be principally used for other dominant sarcomeric gene mutations since the stoichiometry of the sarcomere should be conserved. Whereas the latter idea needs experimental confirmation, the observed suppression of pathologic mRNA species by gene therapy is clearly important. Indeed, we previously showed that, in contrast to homozygous knockout mice that are deficient in $\mathrm{CMyBP}-\mathrm{C}$, the presence of even a low level of cMyBP-C mutants in homozygous and heterozygous $\mathrm{KI}$ mice have a dominant-negative effect on the function of the ubiquitin-proteasome system upon adrenergic stress or aging ${ }^{9-11}$.

Long-term expression of CMyBP-C in the heart was achieved by a single intravenous injection in 1-day-old KI mice using a combination of AAV9, which is the most cardiotropic serotype in rodents $^{21,36}$ and a cardiomyocyte-specific promoter ${ }^{36,37}$. Previous data indicated that combining AAV9 and a chicken minimal truncated TNNT2 promoter gave $>640$-fold higher expression in the heart than in other organs in mice ${ }^{37}$. Here, we used a human minimal truncated TNNT2 promoter that showed restriction of FLAG-cMyBP-C protein expression to the heart 34 wks following systemic administration in vivo, supporting recent data using the same combination of AAV9 and TNNT2 promoter 13 wks after injection ${ }^{38}$. Serial echocardiographic analyses were done by a highly experienced investigator blinded to the genotype or treatment group. It still showed relevant intra- and inter-subject variability over time and, due to multiple group testing (two-way analysis of variance (ANOVA) for groups and time points) and the relatively small number of mice in each group $(n=5-12)$, only reached significant differences between PBS-treated KI and WT. However, separated evaluation at all time points using oneway ANOVA showed significant difference between groups with respect to different parameters, including $\mathrm{LVM} / \mathrm{BW}$ and FAS (Supplementary Table 3). In addition, HW/BW and HW/TL ratios, very robust parameters, revealed clear and significant effects of gene therapy. Furthermore, the dose dependency of the functional improvement and the haemodynamic data all support the conclusion that gene therapy in newborn KI mice partially prevented the disease phenotype. Of note, the effect of $M y b p c 3$ gene therapy was larger than anything reported previously ${ }^{21,22}$, but still remained incomplete, both in terms of molecular and functional restoration. Whereas total $M y b p c 3$ mRNA levels almost reached WT levels in KI treated with the highest dose, protein levels reached maximally $\sim 60 \%$ of WT levels. This incomplete restoration is also revealed by the patchy pattern as shown by immunofluorescence, that is, some cells showed normal staining, others no staining at all. Likely, the WT protein level per ventricular myocytes cannot exceed $100 \%$, whereas mRNA levels $\mathrm{can}^{35}$, resulting in slightly different average mRNA and protein levels after gene transfer. In addition, the stability of the exogenous protein could be slightly altered (reduced translation or accelerated degradation). Finally, some cardiac myocytes may undergo cell division after birth and therefore loose the AAV9 (ref. 39).

Limitations of the study should be noted. First, it showed prevention of a disease phenotype, but no rescue. Yet, we believe that prevention is the more realistic scenario in the patient group we would like to target with the gene therapy approach, namely infants with severe homozygous or compound heterozygous mutations who quickly develop a therapy-resistant form of systolic heart failure ${ }^{14,16-20}$. Second, mouse models including our KI model clearly differ from human HCM in that they show a much milder phenotype, generally only at the homozygous state $^{8,40,41}$. In fact, our KI mice, although likely the best murine HCM model available, have a normal life expectancy and, as shown also in this study, LVH and the decrease in LV contractile function remain stable over time. However, this also means that the maximal therapeutic effect is smaller than expected for large animals or humans with homozygous mutations. Finally, practical issues such as the presence of neutralizing antibodies against AAV9 do not need to be considered in mice, but may constitute a real problem in patients particularly in infants ${ }^{42}$.

In summary, the present study reports the first successful longterm gene therapy of HCM that should be principally applicable to all forms of severe HCM caused by mutations in MYBPC3. The target population for such therapy are newborn children with homozygous or compound heterozygous MYBPC3 mutations who, similar to KI mice, are born relatively normal, but quickly develop severe LV dysfunction and dyspnoea, dying on average within 1 year. We believe that for such children MYBPC3 gene therapy could be a realistic option and we currently work on translating the concept to a large animal model.

\section{Methods}

Animals. The investigation conforms to the guidelines for the care and use of laboratory animals published by the NIH (Publication No. 85-23, revised 1985). The experimental procedures were in accordance with the German Law for the Protection of Animals and accepted by the Ministry of Science and Public Health of the City State of Hamburg, Germany (No. 69/10). KI mice were generated previously ${ }^{8}$, and both KI and WT mice were maintained on a Black Swiss background.

FLAG-Mybpc3 construct. The pGG2-TNNT2-FLAG-Mybpc3 was generated by an exchange of the cytomegalovirus-intron cassette of the pGG2 expression vector with the TNNT2-intron cassette derived from the shuttle plasmid pds-TNNT2-Rluc 
by double digestion with EcoRI/NheI. The full-length FLAG-Mybpc3 cDNA comprising exons 1-34 was amplified by PCR from murine ventricular WT Mybpc3 cDNA with the forward primer including the XhoI restriction site, the ATG followed by the FLAG sequence and the first $20 \mathrm{nt}$ of $M y b p c 3$ exon 1 . The reverse primer contained the last 4 nucleotides of exon 33 plus 8 nucleotides of exon 34, the TGA stop codon followed by the BamHI restriction site. The PCR was done using a touchdown program $\left(70^{\circ} \mathrm{C}-65^{\circ} \mathrm{C}\right)$ with Phusion Hot Start II HighFidelity DNA polymerase and 36 cycles. PCR product and the pGG2-TNNT2 vector were then Xhol/BamHI digested and purified by gel extraction. After ligation, ampicillin-resistant clones were tested by enzymatic digestion for presence of the insert and one positive pGG2-TNNT2-FLAG-Mybpc3 clone was sequenced.

Production and titration of AAV particles. AAV6 pseudotyped vectors were generated by co-transfection of HEK293-AAV cells (Biocat) with the pdsAAVTNNT2-FLAG-Mybpc3 transfer plasmid and the AAV-packaging plasmid pDP6rs, which provides the AAV2 rep and AAV6 cap genes and adenoviral helper functions. AAV9 pseudotyped vectors were generated by triple-transfection of pdsAAV-TNNT2-FLAG-Mybpc3 transfer plasmid with pAAV2/9 and pHelper encoding adenoviral helper functions (Biocat). Generation of recombinant AAV6 and AAV9 particles was carried out as follow. Briefly, $1.5 \times 10^{7}$ HEK293-AAV cells were seeded on $15-\mathrm{cm}$ plates and transfected with polyethylenimine. Three days after transfection cells were collected, washed three times with PBS and resuspended in PBS. Three cycles of freeze-thaw were done before incubation $(1 \mathrm{~h}$ at $37^{\circ} \mathrm{C}$ ) with benzonase (Merk; final concentration $250 \mathrm{U} \mathrm{ml}^{-1}$ ). Cells debris were pelleted and vector-containing lysate were purified by iodixanol step gradients. The genomic titres of AAV6 and AAV9 were determined by qPCR using SYBR Green qPCR Master Mix2 (Fermentas) and an ABI PRISM 7900HT cycler (Applied Biosystem). Vectors were titrated using primers specific for the TNNT2 promoter sequence and a standard curve obtained by serial dilutions of the respective plasmids.

Experiments in neonatal mouse cardiac myocytes. Neonatal mouse cardiac myocytes (NMCMs) were isolated from at least 20 hearts of 1-4-day-old KI mice after an overnight incubation with $0.5 \mathrm{mg} \mathrm{ml}^{-1}$ trypsin followed by repeated digestion with $240 \mathrm{U} \mathrm{ml}^{-1}$ collagenase type II. The isolated cells were then preplated twice to exclude non-muscle cells. NMCMs were finally plated on laminincoated dishes at a density of $10^{5}$ cells $\mathrm{cm}^{-2}$. AAV6-mediated transductions of cardiac myocytes were performed for $30 \mathrm{~min}$ at $37^{\circ} \mathrm{C}$ in suspension prior to plating $\left(4.4 \times 10^{5}\right.$ cells per well) at a multiplicity of infection of 3,000 . Cardiac myocytes were kept in culture for 7 days at $37^{\circ} \mathrm{C}$ and $10 \% \mathrm{CO}_{2}$ prior to collection.

Experiments in mouse EHTs. EHTs were generated from unpurified mouse cardiac cells ${ }^{24}$. In brief, isolated cells were resuspended at a density of $6.8 \times 10^{6}$ cells $\mathrm{ml}^{-1}$ in culture medium containing bovine fibrinogen $\left(5 \mathrm{mg} \mathrm{ml}^{-1}\right)$, aprotinin $\left(2.5 \mu \mathrm{g} \mathrm{ml}^{-1}\right)$ and $10 \%$ Matrigel (BD Bioscience). The reconstitution mix $(97 \mu \mathrm{l} /$ EHT) was mixed with thrombin $\left(3 \mu \mathrm{l} / \mathrm{EHT}, 100 \mathrm{U} \mathrm{ml}^{-1}\right)$ and pipetted into rectangular agarose casting moulds in a 24-well plate. Prior to this pipetting step, silicon racks with four pairs of elastic silicone posts each were placed onto the 24-well plate so that one pair of posts reached from the top the cell-fibrinogenthrombin mix contained in the casting mould. Fibrin polymerization led to a muscle strip around the silicone posts. The racks were then transferred to fresh 24-well plates containing culture medium (Dulbecco's modified Eagle's medium, $10 \%$ horse serum, $2 \%$ chick embryo extract, $1 \%$ penicillin/streptomycin, $10 \mu \mathrm{g} \mathrm{ml}^{-1}$ insulin and $33 \mu \mathrm{g} \mathrm{ml}^{-1}$ aprotinin) and maintained at $21 \%$ oxygen. On day 5 cytosine $\beta$-D-arabinofuranoside $\left(25 \mu \mathrm{g} \mathrm{ml}^{-1}\right)$ was added to the culture medium for $48 \mathrm{~h}$. AAV6 transduction (multiplicity of infection 1,000) was performed directly into the freshly isolated heart cells before adding reconstitution mix components and casting EHTs ${ }^{43,44}$. Contractility measurements were performed on day 19. Briefly, contractions were measured by video-optical recording using a customized software (http://www.ctmv.de), based on figure recognition of the contracting muscle strip in a fully automated manner. Values for average force, contraction and relaxation velocity were calculated with a specific custom-made algorithm.

In vivo AAV9 administration. One-day-old KI mice received different doses of AAV9 (from $1 \times 10^{11}$ to $3 \times 10^{12}$ vg per mouse) or PBS via systemic administration into the temporal vein using a $30-\mathrm{G}$ needle ${ }^{21,22}$.

Echocardiographic analysis. Transthoracic echocardiography was performed using the Vevo 2100 System (VisualSonics, Toronto, Canada). Mice were anaesthetized with isoflurane $(1-2 \%)$ and taped to a warming platform in a supine position. B-mode images were obtained using a MS400 transducer for adult mice and a MS550 transducer for neonatal mice. Images were obtained in a parasternal short and long-axis view and dimensions of the left ventricle were measured in a short-axis view in diastole and systole.
In vivo haemodynamics. Haemodynamic measurements were performed in an open-chest approach in 34-wk-old mice. Mice were anaesthetized with isoflurane (3.5\% for induction, $2 \%$ during the recording), and buprenorphine $\left(0.5 \mathrm{mg} \mathrm{kg}^{-1}\right.$ $\mathrm{BW}$ ) was administered for analgesia. Animals were assured to a warming platform in a supine position. Tracheotomy was performed and mice were artificially ventilated with a rodent ventilator (MiniVent Type 845, Hugo Sachs). The abdomen was opened subxiphoidally. The diaphragm was incised via a transversal subcostal approach and the pericardium was opened. The LV was entered via an apical stab with a 25 -gauge needle, followed by a $1.2-\mathrm{F}$ transonic catheter. After a stabilization period of $5 \mathrm{~min}$ heart rate, left ventricular end-diastolic and end-systolic pressure and LV systolic $\left(\mathrm{d} P / \mathrm{d} t_{\max }\right)$ and diastolic $\left(\mathrm{d} P / \mathrm{d} t_{\min }\right)$ functions were recorded with the Scisense ADVANTAGE System.

RT-PCR and RT-qPCR. Total RNA was isolated from ventricular tissue or EHTs using the SV Total RNA Isolation System Kit (Promega) or Trizol reagent, respectively, according to the manufacturer's instructions. RT was performed from 200 ng RNA from EHTs or pooled RNA (200 ng RNA per sample) using oligo(dT) primers and the SuperScript III kit (Life Technologies). RT-PCR and RT-qPCR were performed with different primer pairs and probes (Supplementary Table 1). Touchdown RT-PCR amplifications $\left(65-60^{\circ} \mathrm{C}\right.$ or $60-55^{\circ} \mathrm{C}$ depending on primer pairs) were performed using AmpliTaq Gold Polymerase (Applied Biosystem) in a total volume of $20 \mu \mathrm{l}$ and 35 cycles. PCR products were visualized on $2 \%$ agarose gel. RT-qPCR for total Mybpc3 mRNA level and hypertrophic markers were performed with SYBR Green qPCR Master Mix 2 (Fermentas). WT and mutant $M y b p c 3$ mRNA levels were determined with specific hydrolysing probes. Data were normalized to $\mathrm{G} \alpha \mathrm{S}$.

Western blot analysis. For western blots analysis crude proteins were extracted from cultured cardiac myocytes or tissues, separated on 10\% SDS-polyacrylamide (29:1) mini-gels (Bio-Rad) and electrotransferred on polyvinylidene difluoride or nitrocellulose membranes. Primary antibodies were directed against the FLAG epitope (1:5,000; Sigma), the MyBP-C motif (1:1,000), lamin A/C (1:2,000; Santa Cruz), or GAPDH (1:2,000; HyTest). Secondary antibodies were anti-mouse (1:20,000; Dianova) or anti-rabbit (1:6,000; Sigma) peroxidase-conjugated. Proteins were visualized using the Super Signal West Dura detection reagent (Thermo Scientific) and signals were detected with the ChemiGenius ${ }^{2}$ Bio Imaging System. Full western blots from tissue samples are displayed in Supplementary Fig. 1.

Immunofluorescence analysis. For immunofluorescence analysis, AAV6-transduced KI NMCMs were cultured 7 days on glass coverslips prior to fixation with methanol/acetone $(20 / 80)$ at $-20^{\circ} \mathrm{C}$. For immunofluorescence of 10 - $\mu \mathrm{m}$-thick cardiac sections, hearts were rinsed in $\mathrm{KCl}$ solution and embedded in Tissue Tek ${ }^{\mathbb{R}}$. After permeabilization, cells and cardiac sections were stained with primary antibodies, the anti-FLAG (1:800, Sigma), anti-cMyBP-C directed against the MyBP-C motif (1:500) and anti- $\alpha$-actinin (1:800, Sigma). Secondary antibodies were the anti-mouse IgG Alexa 488-conjugated, 1:800 and anti-rabbit IgG Alexa 546-conjugated, 1:800 (Molecular Probes). Nuclei were stained with DRAQ5 (1:1,000; Biostatus). Confocal images were acquired with a Zeiss LSM 710 system using a Zeiss Axiovert microscope.

Statistical analysis. Data were expressed as mean \pm s.e.m. Statistical analyses were performed by one-way or two-way ANOVA followed by Dunnett's or Bonferroni's post-test, and by the Student's $t$-test as indicated in figure legends or text, using the commercial software GraphPad Prism5 (Software Inc.). A value of $P<0.05$ was considered statistically significant.

\section{References}

1. Maron, B. J. et al. Prevalence of hypertrophic cardiomyopathy in a general population of young adults. Echocardiographic analysis of 4111 subjects in the CARDIA Study. Coronary Artery Risk Development in (Young) Adults. Circulation 92, 785-789 (1995)

2. Behrens-Gawlik, V., Mearini, G., Gedicke-Hornung, C., Richard, P. \& Carrier, L. MYBPC3 in hypertrophic cardiomyopathy: from mutation identification to RNA-based correction. Pflugers. Arch. 466, 215-223 (2014).

3. Schlossarek, S., Mearini, G. \& Carrier, L. Cardiac myosin-binding protein C in hypertrophic cardiomyopathy: mechanisms and therapeutic opportunities J. Mol. Cell. Cardiol. 50, 613-620 (2011).

4. van Dijk, S. J. et al. Cardiac myosin-binding protein C mutations and hypertrophic cardiomyopathy: haploinsufficiency, deranged phosphorylation, and cardiomyocyte dysfunction. Circulation 119, 1473-1483 (2009).

5. van Dijk, S. J. et al. Contractile dysfunction irrespective of the mutant protein in human hypertrophic cardiomyopathy with normal systolic function. Circ. Heart Fail. 5, 36-46 (2012).

6. Marston, S., Copeland, O., Gehmlich, K., Schlossarek, S. \& Carrier, L. How do MYBPC3 mutations cause hypertrophic cardiomyopathy? J. Muscle Res. Cell Motil. 33, 75-80 (2012). 
7. Meurs, K. M. et al. A cardiac myosin binding protein C mutation in the Maine Coon cat with familial hypertrophic cardiomyopathy. Hum. Mol. Genet. 14, 3587-3593 (2005).

8. Vignier, N. et al. Nonsense-mediated mRNA decay and ubiquitin-proteasome system regulate cardiac myosin-binding protein $\mathrm{C}$ mutant levels in cardiomyopathic mice. Circ. Res. 105, 239-248 (2009).

9. Schlossarek, S. et al. Defective proteolytic systems in Mybpc3-targeted mice with cardiac hypertrophy. Basic Res. Cardiol. 107, 1-13 (2012).

10. Schlossarek, S., Frey, N. \& Carrier, L. Ubiquitin-proteasome system and hereditary cardiomyopathies. J. Mol. Cell Cardiol. 71, 25-31 (2014).

11. Schlossarek, S. et al. Adrenergic stress reveals septal hypertrophy and proteasome impairment in heterozygous Mybpc3-targeted knock-in mice. J. Muscle Res. Cell Motil. 33, 5-15 (2012).

12. Elliott, P. et al. Classification of the cardiomyopathies: a position statement from the European Society Of Cardiology Working Group on Myocardial and Pericardial Diseases. Eur. Heart J. 29, 270-276 (2008).

13. Maron, B. J., Doerer, J. J., Haas, T. S., Tierney, D. M. \& Mueller, F. O. Sudden deaths in young competitive athletes: analysis of 1866 deaths in the United States, 1980-2006. Circulation 119, 1085-1092 (2009).

14. Marziliano, N. et al. A case of compound mutations in the MYBPC3 gene associated with biventricular hypertrophy and neonatal death. Neonatology 102, 254-258 (2012).

15. El-Saiedi, S. A., Seliem, Z. S. \& Esmail, R. I. Hypertrophic cardiomyopathy: prognostic factors and survival analysis in 128 Egyptian patients. Cardiol. Young 1-7 (2013).

16. Dellefave, L. M. et al. Sarcomere mutations in cardiomyopathy with left ventricular hypertrabeculation. Circ. Cardiovasc. Genet. 2, 442-449 (2009).

17. Lekanne Deprez, R. H. et al. Two cases of severe neonatal hypertrophic cardiomyopathy caused by compound heterozygous mutations in the MYBPC3 gene. J. Med. Genet. 43, 829-832 (2006).

18. Xin, B., Puffenberger, E., Tumbush, J., Bockoven, J. R. \& Wang, H. Homozygosity for a novel splice site mutation in the cardiac myosin-binding protein $\mathrm{C}$ gene causes severe neonatal hypertrophic cardiomyopathy. Am. J. Med. Genet. A 143A, 2662-2667 (2007).

19. Richard, P. et al. Hypertrophic Cardiomyopathy: distribution of disease genes, spectrum of mutations and implications for molecular diagnosis strategy. Circulation 107, 2227-2232 (2003).

20. Schaefer, E. et al. Next-generation sequencing (NGS) as a fast molecular diagnosis tool for left ventricular noncompaction in an infant with compound mutations in the MYBPC3 gene. Eur. J. Med. Genet. 57, 129-132 (2014).

21. Gedicke-Hornung, C. et al. Rescue of cardiomyopathy through U7snRNAmediated exon skipping in Mybpc3-targeted knock-in mice. EMBO Mol. Med. 5, 1128-1145 (2013).

22. Mearini, G. et al. Repair of Mybpc3 mRNA by 5'-trans-splicing in a mouse model of hypertrophic cardiomyopathy. Mol. Ther. Nucleic Acids 2, e102 (2013).

23. Olivotto, I. et al. Myofilament protein gene mutation screening and outcome of patients with hypertrophic cardiomyopathy. Mayo. Clin. Proc. 83, 630-638 (2008).

24. Stohr, A. et al. Contractile abnormalities and altered drug response in engineered heart tissue from Mybpc3-targeted knock-in mice. J. Mol. Cell. Cardiol. 63, 189-198 (2013).

25. Dominguez, E. et al. Intravenous scAAV9 delivery of a codon-optimized SMN1 sequence rescues SMA mice. Hum. Mol. Genet. 20, 681-693 (2011).

26. Kaye, D. M. et al. Percutaneous cardiac recirculation-mediated gene transfer of an inhibitory phospholamban peptide reverses advanced heart failure in large animals. J. Am. Coll. Cardiol. 50, 253-260 (2007).

27. Pleger, S. T. et al. Cardiac AAV9-S100A1 gene therapy rescues post-ischemic heart failure in a preclinical large animal model. Sci. Transl. Med. 3, 92ra64 (2011).

28. Jessup, M. et al. Calcium upregulation by percutaneous administration of gene therapy in cardiac disease (CUPID): a phase 2 trial of intracoronary gene therapy of sarcoplasmic reticulum $\mathrm{Ca} 2+-$ ATPase in patients with advanced heart failure. Circulation 124, 304-313 (2011).

29. Spoladore, R., Maron, M. S., D’Amato, R., Camici, P. G. \& Olivotto, I. Pharmacological treatment options for hypertrophic cardiomyopathy: high time for evidence. Eur. Heart J. 33, 1724-1733 (2012).

30. Frey, N., Luedde, M. \& Katus, H. A. Mechanisms of disease: hypertrophic cardiomyopathy. Nat. Rev. Cardiol. 9, 91-100 (2012).

31. Jiang, J., Wakimoto, H., Seidman, J. G. \& Seidman, C. E. Allele-specific silencing of mutant Myh6 transcripts in mice suppresses hypertrophic cardiomyopathy. Science 342, 111-114 (2013).

32. Mamidi, R., Li, J., Gresham, K. S. \& Stelzer, J. E. Cardiac myosin binding protein-C: a novel sarcomeric target for gene therapy. Pflugers. Arch. 466, 225-230 (2014).
33. Wu, Z., Yang, H. \& Colosi, P. Effect of genome size on AAV vector packaging. Mol. Ther. 18, 80-86 (2010).

34. Grieger, J. C. \& Samulski, R. J. Packaging capacity of adeno-associated virus serotypes: impact of larger genomes on infectivity and postentry steps. J. Virol. 79, 9933-9944 (2005).

35. James, J. et al. Transgenic over-expression of a motor protein at high levels results in severe cardiac pathology. Transgenic Res. 8, 9-22 (1999).

36. Bish, L. T. et al. Adeno-associated virus (AAV) serotype 9 provides global cardiac gene transfer superior to AAV1, AAV6, AAV7, and AAV8 in the mouse and rat. Hum. Gene Ther. 19, 1359-1368 (2008).

37. Prasad, K. M., Xu, Y., Yang, Z., Acton, S. T. \& French, B. A. Robust cardiomyocyte-specific gene expression following systemic injection of AAV: in vivo gene delivery follows a Poisson distribution. Gene Ther. 18, 43-52 (2011).

38. Werfel, S. et al. Rapid and highly efficient inducible cardiac gene knockout in adult mice using AAV-mediated expression of Cre recombinase. Cardiovasc. Res. 104, 15-23 (2014)

39. Li, F., Wang, X., Capasso, J. M. \& Gerdes, A. M. Rapid transition of cardiac myocytes from hyperplasia to hypertrophy during postnatal development. $J$. Mol. Cell. Cardiol. 28, 1737-1746 (1996).

40. Harris, S. P. et al. Hypertrophic cardiomyopathy in cardiac myosin binding protein-C knockout mice. Circ. Res. 90, 594-601 (2002).

41. Carrier, L. et al. Asymmetric septal hypertrophy in heterozygous cMyBP-C null mice. Cardiovasc. Res. 63, 293-304 (2004).

42. Calcedo, R. et al. Adeno-associated virus antibody profiles in newborns, children, and adolescents. Clin. Vaccine Immunol. 18, 1586-1588 (2011).

43. Friedrich, F. W. et al. Evidence for FHL1 as a novel disease gene for isolated hypertrophic cardiomyopathy. Hum. Mol. Genet. 21, 3237-3254 (2012).

44. Crocini, C. et al. Impact of ANKRD1 mutations associated with hypertrophic cardiomyopathy on contraction parameters of engineered heart tissue. Basic Res. Cardiol. 108, 349 (2013).

\section{Acknowledgements}

We would like to thank Evelyn Bendrat and Michaela Miehe (UKE-HEXT, Hamburg, Germany), and Mareen Welzel and Andreas Jungmann (University Hospital Heidelberg, Germany) for their technical support in AAV production. We also thank June Uebeler (UKE-Pharmacology, Hamburg, Germany) for help in cardiac myocytes isolation. We thank Christian Witt (University of Heidelberg, Germany) for the anti-cMyBP-C antibody. We also thank Nicolas Vignier (Paris) for fruitful discussions. This work was supported by the DZHK (German Centre for Cardiovascular Research), the German Ministry of Research and Education (BMBF) and the Association Institut de Myologie (Paris).

\section{Author contributions}

G.M. designed and performed experiments (cloning, immunofluorescence and molecular biology), wrote parts of the first draft of the manuscript, supervised D.S., E.K., J.M.-F. and A.D.D.S. performed experiments in cardiac myocytes (isolation and transduction), western blots, and assisted the AAV administration in mice. B.G. performed experiments in mice in vivo (administration of AAV, echocardiography and haemodynamics and echocardiography analysis). F.W. performed experiments in mice in vivo (echocardiography, haemodynamics and evaluation). E.K. did molecular biology experiments (cloning, RT-PCR and RT-qPCR analysis). J.M.-F., A.S., A.D. and P.J.M.W. performed experiments in EHTs and analysis. S.S. performed western blots and managed the mouse cohorts. I.B., H.A.K. and O.J.M. supervised productions of AAV. O.J.M., T.V. and T.E. critically discussed the manuscript. L.C. designed and supervised the entire study, obtained fundings and wrote the manuscript. All authors critically discussed the results, and reviewed and approved the manuscript before submission.

\section{Additional information}

Supplementary Information accompanies this paper at http://www.nature.com/ naturecommunications

Competing financial interests: L.C., T.E., T.V., O.J.M., G.M., D.S. and J.M.-F. are co-authors of a provisional European Patent Application No. EP13164212, filed 17 April 2013, 'Gene-therapy vectors for treating cardiomyopathy', followed by a provisional international Patent Application No. PCT/EP2014/057984, 17 April 2014. The remaining authors declare no competing financial interests.

Reprints and permission information is available online at http://npg.nature.com/ reprintsandpermissions/

How to cite this article: Mearini, G. et al. Mybpc3 gene therapy for neonatal cardiomyopathy enables long-term disease prevention in mice. Nat. Commun. 5:5515 doi: $10.1038 /$ ncomms6515 (2014) 